\title{
Field dependent surface resistance of niobium on copper cavities
}

\author{
T. Junginger ${ }^{*}$ \\ CERN, Geneva, Switzerland
}

(Received 4 December 2014; published 20 July 2015)

\begin{abstract}
The surface resistance $R_{\mathrm{S}}$ of superconducting cavities prepared by sputter coating a niobium film on a copper substrate increases significantly stronger with the applied rf field compared to cavities of bulk material. A possible cause is that the thermal boundary resistance between the copper substrate and the niobium film induces heating of the inner cavity wall, resulting in a higher $R_{\mathrm{S}}$. Introducing helium gas in the cavity, and measuring its pressure as a function of applied field allowed to conclude that the inner surface of the cavity is heated up by less than $120 \mathrm{mK}$ when $R_{\mathrm{S}}$ increases with $E_{\text {acc }}$ by $100 \mathrm{n} \Omega$. This is more than one order of magnitude less than what one would expect from global heating. Additionally, the effects of cooldown speed and low temperature baking have been investigated in the framework of these experiments. It is shown that for the current state of the art niobium on copper cavities there is only a detrimental effect of low temperature baking. A fast cooldown results in a lowered $R_{\mathrm{S}}$.
\end{abstract}

DOI: 10.1103/PhysRevSTAB.18.072001

PACS numbers: 29.20.-c, 74.25.nn, 74.78.-w

\section{INTRODUCTION}

Superconducting cavities prepared by coating a micrometer thick niobium film on a copper substrate are currently used at CERN in the LHC and for the HIE-Isolde project [1]. The operation temperature here is $4.5 \mathrm{~K}$, where this technology enables a lower surface resistance than cavities prepared from bulk material, caused by the lower residual resistance ratio (RRR). The Bardeen Cooper Schrieffer (BCS) surface resistance is minimal if the electron mean free path is approximately as long as the BCS coherence length [2]. This condition is met for a RRR of about 10 in case of niobium, a typical value obtained for films on cavities prepared by magnetron sputtering [3]. Other advantages of $\mathrm{Nb} / \mathrm{Cu}$ cavities are lower material cost, no need for shielding the Earth's magnetic field, and enhanced thermal stability avoiding quenches [4]. For quarter-wave resonators, cryostat design is facilitated since these copper structures can be cooled by conduction [1].

Despite these advantages, the $\mathrm{Nb} / \mathrm{Cu}$ technology is currently not considered for accelerators requiring highest accelerating gradient $E_{\text {acc }}$ like the ILC, or lowest surface resistance $R_{\mathrm{S}}$ at temperatures of $2 \mathrm{~K}$ and below, especially for high duty cycle and continuous wave applications. The reason is that $R_{\mathrm{S}}$ increases strongly with $E_{\mathrm{acc}}$. The origin of this field dependent surface resistance has been the subject of many studies in the past $[3,5,6]$, but is still far from being fully understood. The film thickness of about $1.5 \mu \mathrm{m}$ is

\footnotetext{
*Present address: TRIUMF, Vancouver, Canada. tobi@triumf.ca

Published by the American Physical Society under the terms of the Creative Commons Attribution 3.0 License. Further distribution of this work must maintain attribution to the author(s) and the published article's title, journal citation, and DOI.
}

large compared to the London penetration depth $\lambda_{\mathrm{L}}$ of about $32 \mathrm{~nm}$. Therefore differences in the performance (apart from thermal conductivity issues) should be largely due to the manufacturing procedure and the resulting surface structure. No single dominant source can be expected, thus several hypotheses need to be addressed individually to identify their origin and possibly reduce their extent. In this paper the effect of a potential temperature difference between the inner cavity surface and the helium bath, induced by rf heating, is investigated. The influence on the rf performance of the cooldown speed and low temperature baking are addressed.

An issue in superconducting cavities is the possibility that the thermal impedance of the cavity wall leads to an increased temperature of the inner surface exposed to the rf, resulting in an increased surface resistance [6]. For bulk niobium cavities it has been shown that this effect at least contributes to the field dependent surface resistance [7]. For $\mathrm{Nb} / \mathrm{Cu}$ cavities the problem is even more complex, since the thermal impedance across the film/substrate interface is unknown and adds to the total thermal conductivity. Here a method previously tried in [6] to directly measure the inner surface temperature of a superconducting cavity is investigated in detail.

Thermal cycling has been studied in detail by several laboratories on bulk niobium [8,9] and also on nitrogen doped niobium cavities [9]. It is however still unclear, what the ideal cooldown procedure is and if it depends on the material treatment of the cavity. Another open question is how much magnetic flux created by thermal currents influences $R_{\mathrm{S}}$, which could be the reason for differences observed between vertical and horizontal test results [8]. Studies on $\mathrm{Nb} / \mathrm{Cu}$ cavities are not only useful to push their performance, but also to obtain further information about the mechanism of thermal cycling in general, since $\mathrm{Nb} / \mathrm{Cu}$ 
cavities are a lot less sensitive to ambient magnetic fields [3]. At CERN such studies have been carried out using the Quadrupole Resonator $[10,11]$ on a $\mathrm{Nb} / \mathrm{Cu}$ sample prepared by electron cyclotron resonance (ECR) sputtering [12] and on $\mathrm{HIE}$ Isolde $\mathrm{Nb} / \mathrm{Cu}$ quarter wave resonators [13]. In this paper the first study on elliptical $\mathrm{Nb} / \mathrm{Cu}$ cavities is presented. Results will be compared with the references given above and an advice for a cooldown procedure of cryomodules equipped with $\mathrm{Nb} / \mathrm{Cu}$ cavities will be given.

Low temperature in situ baking is used for bulk niobium cavities to shift the so-called $Q$-drop (exponential increase of the surface resistance at high field) to larger values enabling economical high gradient operation [14]. In the medium field region above $10 \mathrm{MV} / \mathrm{m} \mathrm{Nb} / \mathrm{Cu}$ cavities also exhibit an exponential increase of $R_{\mathrm{S}}$ with $E_{\text {acc }}$. One might therefore argue that the origin for this is the same as for the $Q$-drop of bulk niobium cavities. To test this hypothesis the cavity used in this study was tested under the same conditions before and after a $150^{\circ} \mathrm{C}$ bakeout.

\section{EXPERIMENTAL SETUP AND PROCEDURE}

A micrometer thick niobium film has been deposited by high-power impulse magnetron sputtering (HIPIMS) technology on the inner surface of an elliptical single-cell TESLA type [15] $1.3 \mathrm{Ghz}$ copper cavity produced by spinning and electropolishing. The important cavity parameters for the studies presented here are found in Table I. They relate the peak magnetic and electric fields to the accelerating gradient $E_{\text {acc }}$ and the unloaded quality factor $Q_{0}$ to the surface resistance $R_{\mathrm{S}}$. In the following always $E_{\text {acc }}$ and $R_{\mathrm{S}}$ will be used. The HIPIMS sputtering technique for superconducting cavities is under investigation at CERN since 2010 [16]. Literature shows that better film morphology, denser and smoother films, can be achieved compared to standard de magnetron sputtering (dcMS) coating technology [17]. The cavity analyzed here has been coated with a peak current of 200 A (current density of $2 \mathrm{~A} / \mathrm{cm}^{2}$ ), a potential of $570 \mathrm{~V}$, an average current of $2.6 \mathrm{~A}$ with a pulse width of $200 \mu$ s and a frequency of $106 \mathrm{~Hz}$ [16]. Preceding sample studies have motivated these parameter choices [18]. A detailed description of the coating system as well as plasma and surface characterization is reported elsewhere [18]. So far the rf cavity results show systematically an increase of $R_{\mathrm{S}}$ with $E_{\text {acc }}$, similar to what has been observed in the past for cavities coated by dcMS [3].

TABLE I. Parameters of TESLA type cavities [15].

\begin{tabular}{lc}
\hline \hline Geometry Factor $\mathrm{G}$ & $270 \Omega$ \\
$E_{\text {peak }} / E_{\text {acc }}$ & 2.0 \\
$B_{\text {peak }} / E_{\text {acc }}$ & $4.26 \mathrm{mT} /(\mathrm{MV} / \mathrm{m})$ \\
\hline \hline
\end{tabular}

For the vertical cold tests the cavity has been equipped with an input coupler with a $Q_{\text {ext }}=2 \times 10^{9}$ and a pick-up antenna with $Q_{\text {pick-up }}=3 \times 10^{11}$. The external coupling $Q_{\text {ext }}$ was chosen to obtain critical coupling at $1.8 \mathrm{~K}$ and $E_{\text {acc }} \approx 15 \mathrm{MV} / \mathrm{m}$. RF measurements were performed using the phase locked loop technique [19]. Temperature sensors were mounted above and below the cavity cell to measure the temperature and its gradient during the phase transition from the normal to the superconducting state. The vertical bath cryostat has been equipped with a mu-metal shield to reduce the ambient magnetic field to about $3 \mu \mathrm{T}$. Helium was transferred using standard dewars, pressurized with helium gas. For the initial cooldown the gas overpressure was set to about 200 mbar. During this first helium transfer the dewar was emptied when the cavity temperature was about $50 \mathrm{~K}$. This yielded a uniform cavity temperature. The temperature gradient at the phase transition was the lowest of all cooldowns performed, see Fig. 1.

In total the cavity was cooled down five times. Each cycle started with a measurement of $R_{\mathrm{S}}$ as a function of $E_{\text {acc }}$ at $4.2 \mathrm{~K}$. Then the cavity was further cooled down to $1.8 \mathrm{~K}$ by pumping the helium bath to 16.5 mbar. This process takes several hours, which is slow enough to take several measurements of $R_{\mathrm{S}}$ at $E_{\mathrm{acc}}=1 \mathrm{MV} / \mathrm{m}$. After the initial cooldown the cavity was warmed up twice above $T_{\mathrm{c}}$ and cooled down again. To ensure that the whole cavity was in the normal conducting state, before the helium transfer was restarted, the temperature on the top and the bottom of the cell were constantly monitored and recorded. Additionally the quality factor was measured with a network analyzer. The maximum temperature the cavity reached in these cycles never exceeded $20 \mathrm{~K}$. For the first thermal cycle the

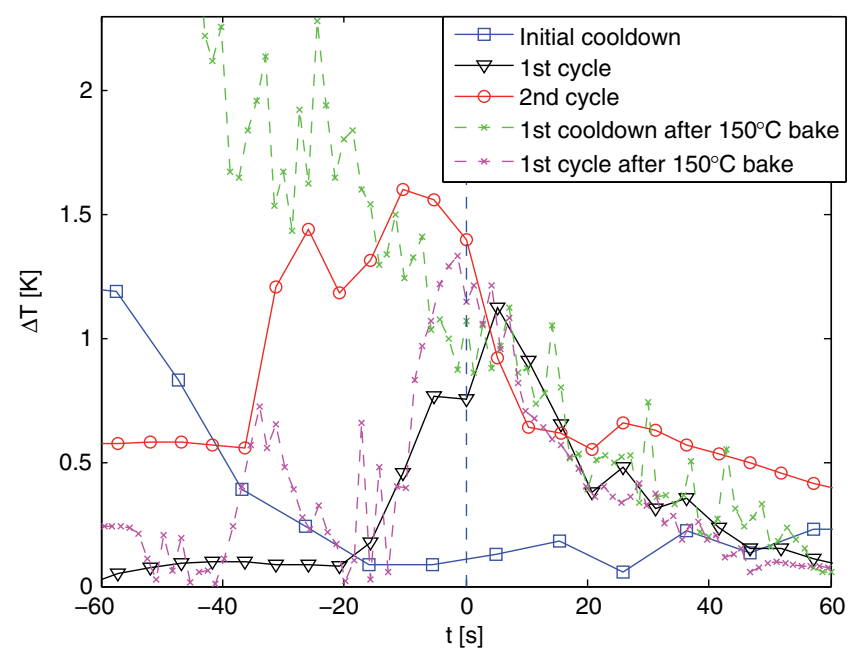

FIG. 1. Temperature gradient along the cell from one minute before until one minute after the phase transition for five cooldowns. 
overpressure was set relatively high to about 250 mbar on the helium supply dewar, while for the second cycle the overpressure was set to only $50 \mathrm{mbar}$ to reduce the cooldown speed. Figure 1 displays the temperature difference $\Delta T$ between the upper and the lower sensor from one minute before until one minute after the lower sensor displayed a value below $T_{\mathrm{c}}$. The upper sensor showed systematically higher values so $\Delta T=T_{\text {up }}-T_{\text {down }}$, where $T_{\text {up }}$ and $T_{\text {down }}$ are the temperature recorded from the upper and the lower sensor at the same time. Temperatures were recorded about every $4 \mathrm{~s}$. The cooldown speed is defined as $T_{\text {down }}\left(t_{-1}\right)-T_{\text {down }}\left(t_{1}\right) /\left(T_{1}-T_{-1}\right)$, where $t_{1}$ and $t_{-1}$ are the times just before and after the phase transition. The cooldown speed was measured to be about $70 \mathrm{mK} / \mathrm{s}$ for the initial cooldown and the second cycle and $230 \mathrm{mK} / \mathrm{s}$ for the first cycle.

After the initial cooldown several additional scans of $R_{\mathrm{S}}$ vs $E_{\text {acc }}$ were performed just below and above the lambda point of helium at $2.17 \mathrm{~K}$. During the warmup to room temperature the frequency shift was measured as a function of temperature to derive the penetration depth. For this, the liquid helium was almost completely evaporated, so that the whole cavity was only cooled by helium gas. A few liters left on the bottom of the cryostat allowed obtaining a constant temperature along the cavity by evaporating some of the liquid. For a precise measurement pressure control is extremely crucial. A PID-controlled butterfly valve was used to pump the bath pressure to 80 mbar. A stability better than 0.02 mbar was achieved during the whole measurement. The pressure was in fact so stable that no frequency change, within the $1 \mathrm{~Hz}$ resolution of the network analyzer, was detectable. The total frequency shift between 4 and $9 \mathrm{~K}$ was about $5 \mathrm{kHz}$. A source of a systematic error is the thermal expansion of copper. Using literature values from [20] it was calculated that the frequency shift due to thermal expansion in the temperature range between 4 and $9 \mathrm{~K}$ is about $70 \mathrm{~Hz}$, two orders of magnitude smaller than the frequency shift caused by the penetration depth change. After complete warmup the cavity was in situ baked at $150{ }^{\circ} \mathrm{C}$ for $50 \mathrm{~h}$. In the following rf tests only one thermal cycle with a fast cooldown has been carried out. The speed was $110 \mathrm{mK} / \mathrm{s}$ for the initial cooldown and $330 \mathrm{mK} / \mathrm{s}$ for the thermal cycle, after which an additional measurement was performed. Helium gas was introduced in the cavity. At $1.8 \mathrm{~K}$ its pressure was $p_{\mathrm{RT}}=5 \times 10^{-5} \mathrm{mbar}$ measured at the top flange connection of the cryostat, which corresponds, according to the thermal transpiration law

$$
p_{\mathrm{cav}}=p_{\mathrm{RT}} \sqrt{\frac{T_{\mathrm{cav}}}{T_{\mathrm{RT}}}}
$$

to a cavity pressure $p_{\text {cav }}=4 \times 10^{-6} \mathrm{mbar}$, where $T_{\text {cav }}$ and $T_{\mathrm{RT}}$ are the cavity and the room temperature,

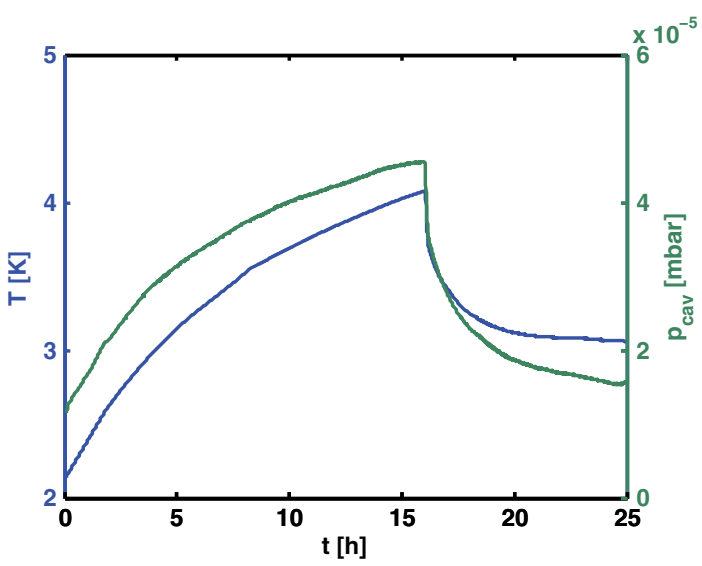

FIG. 2. Temperature and pressure in the cavity during warmup and following cooldown, measured as a function of time.

respectively. The surface resistance was once again measured as a function of $E_{\text {acc }}$, now with the helium gas inside the cavity. To test whether the temperature of the cavity's inner surface increases with $E_{\text {acc }}$ the helium pressure was recorded. In order to calibrate the pressure to temperature values during the following warmup both quantities were recorded. A clear correlation was found, see Fig. 2. To verify that the pressure increase was not caused by a leak introducing just more helium gas with time the helium bath was pumped down again. Once more a clear correlation between $p$ and $T$ was found, see Fig. 2.

For the cooldown lower pressures were measured for the same temperatures. Probably, by pumping on the helium bath the evaporated gas cooled down the upper flange of the cryostat to which the pressure sensor is connected. Now, $T_{\mathrm{RT}}$ is overestimated, yielding with Eq. (1) an underestimated $p_{\text {cav }}$. For the calibration of $p_{\text {cav }}$ with respect to $T$ only the values obtained during warmup were used. These have been recorded directly after the $R_{\mathrm{S}}$ and $p_{\text {cav }}$ vs $E_{\text {ac }}$ measurement. The goal of this calibration was to obtain an upper limit for the heating of the inner cavity surface by $\mathrm{rf}$ as a function of accelerating gradient. Taking the data obtained during the cooldwon one would derive a lower temperature change for the same pressure change. Taking the data of the warmup is therefore better suited to define an upper limit. The cavity pressure $p_{\text {cav }}$ as derived by Eq. (1) as a function of $T$ and a linear fit to the data are displayed in Fig. 3. The physical mechanism, which is responsible for the pressure increase as a function of temperature involves temperature dependent adsorption on the cavity wall and expansion of the nonadsorbed gas with temperature. The pressure of about $p_{\text {cav }}=4 \times 10^{-6}$ mbar was chosen to be high enough that the surface was saturated with helium and a temperature increase results mainly in an increase of the pressure of the nonabsorbed gas. Based on 


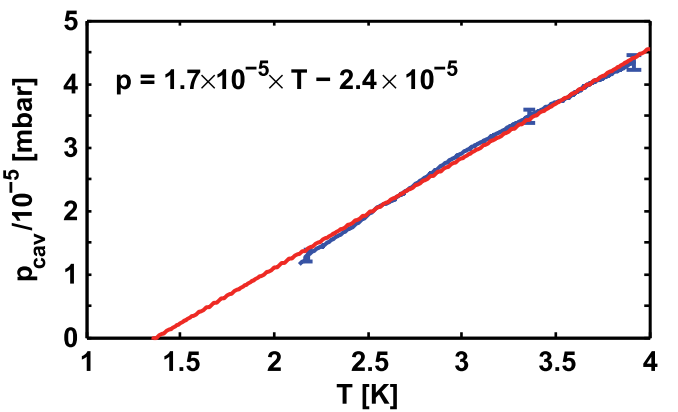

FIG. 3. Cavity pressure as a function of temperature measured during warmup. The blue curve represents measurements obtained every $10 \mathrm{~s}$, while the red line shows a linear fit to the data.

data from [21] it can be estimated that at the pressure used, only a fraction of about $10 \%$ of the gas can be absorbed by the cold cavity surface. An even higher pressure has been tried, but resulted in strong discharging at a few $\mathrm{MV} / \mathrm{m}$ making the measurement impossible. In case of a lower pressure no change of $p$ with $E_{\text {acc }}$ was detectable.

Having a saturated cold surface is important for comparing the pressure increase caused by warming of the helium bath for calibration to the pressure increase due to heating from rf. For example, if one would heat up by rf only the cavity cell releasing gas from its surface it could be adsorbed by the nonsaturated cutoff tubes remaining at bath temperature. In this case a warming of the cavity cell could mainly result in an increase of the adsorbed gas on the cutoff tube surfaces. The linear dependence measured between $p$ and $T$ (Fig. 3) suggests that the ideal gas equation is indeed applicable and the surfaces were well saturated.

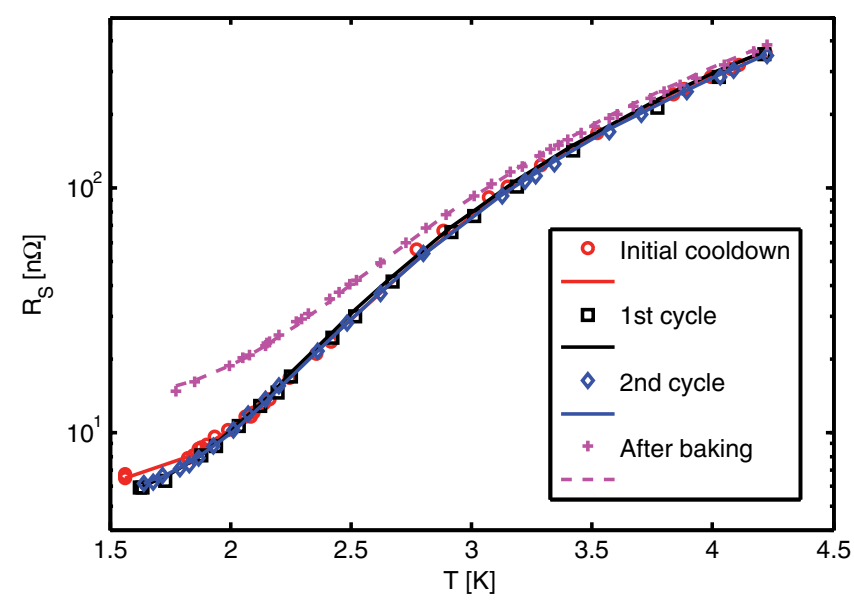

FIG. 4. Surface resistance $R_{\mathrm{S}}$ of a $1.3 \mathrm{GHz} \mathrm{Nb} / \mathrm{Cu}$ cavity as a function of temperature measured after initial cooldown, two following thermal cycles above $T_{\mathrm{c}}$ and after baking.

\section{RESULTS}

\section{A. Low field surface resistance}

For each cooldown cycle the surface resistance was measured as a function of temperature at $E_{\text {acc }}=1 \mathrm{MV} / \mathrm{m}$, see Fig. 4. The data has been fitted to BCS theory using the WinSuperFit software based on calculations from Halbritter [22]. As input parameters the critical temperature $T_{\mathrm{c}}=9.41 \mathrm{~K}$, the London penetration depth for infinite mean free path $\lambda_{\mathrm{L}}=32 \mathrm{~nm}$ and the BCS coherence length $\xi_{0}=39 \mathrm{~nm}$ were used. The former quantity was obtained from frequency shift measurements (see below), while the latter two were taken from the literature [2]. The critical temperature is higher than literature values for bulk niobium, which is typically found for niobium on copper films and can be related to stress in the film [3]. Three parameters were varied in a least squares fit, see Table II. The smallest residual resistance $R_{\text {res }}$ was obtained for the fastest cooldown. The effect is however relatively small. The low temperature bakeout yielded an increase of $R_{\text {res }}$ by several $\mathrm{n} \Omega$. The mean free path could not be accurately determined from $R_{\mathrm{S}}$ vs $T$, because it was not known whether the mean free path is larger or shorter than the coherence length. For thin film cavities this is not obvious, especially not after low temperature baking. From a measurement of the frequency shift, see Fig. 5, however, one can determine $l$ independently even though it has to be noted that here the information depth is different (several $100 \mathrm{~nm}$ ) than for the $R_{\mathrm{S}}$ vs $T$ measurement (about $50 \mathrm{~nm}$ ). From a least squares fit to the GorterCasimir expression [23]

$$
\lambda(T)=\frac{\lambda_{0}}{\sqrt{1-\left(\frac{T}{T_{\mathrm{c}}}\right)^{4}}}
$$

$\lambda_{0}$ and $T_{\mathrm{c}}$ are derived. From $\lambda_{0}$ the electron mean free path $l$ can be obtained from an expression found by Pippard [24]:

TABLE II. Fit parameters obtained from low field surface resistance and penetration depth change measurement.

\begin{tabular}{lcccc}
\hline \hline & $\begin{array}{c}\text { initial } \\
\text { cooldown }\end{array}$ & $\begin{array}{c}\text { 1st } \\
\text { cycle }\end{array}$ & $\begin{array}{c}\text { 2nd } \\
\text { cycle }\end{array}$ & $\begin{array}{c}\text { after } \\
\text { baking }\end{array}$ \\
\hline$\Delta /\left(k_{\mathrm{b}} T_{\mathrm{c}}\right)$ & $1.96 \pm 0.03$ & $1.90 \pm 0.05$ & $1.93 \pm 0.01$ & $1.89 \pm 0.01$ \\
$R_{\mathrm{Res}}$ in $\mathrm{n} \Omega$ & $6.1 \pm 0.2$ & $5.2 \pm 0.2$ & $5.5 \pm 0.2$ & $13.7 \pm 0.4$ \\
$l$ in nm from & $85 \pm 40$ & $25 \pm 120$ & $25 \pm 120$ & $27 \pm 130$ \\
$\quad \mathrm{R} \mathrm{vs} \mathrm{T}$ & & & & \\
$\lambda_{0}$ in nm & & & $46 \pm 4$ & $78 \pm 1$ \\
$l$ in nm & & & $58 \pm 4$ & $12 \pm 1$ \\
$\quad$ from $\Delta \lambda$ & - & - & $9.41 \pm 0.05$ & $9.41 \pm 0.02$ \\
$T_{\mathrm{c}}$ & - &
\end{tabular}




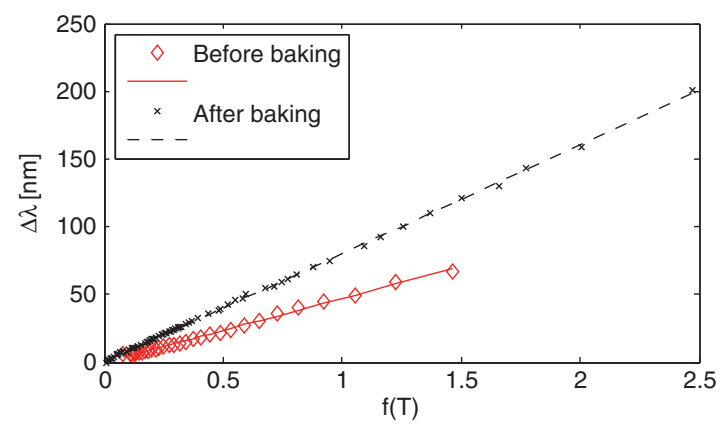

FIG. 5. Penetration depth change as a function of $f(T)=1 /$ $\sqrt{1-\left(\frac{T}{T_{\mathrm{c}}}\right)^{4}}-1$ before and after baking.

$$
\lambda(l)=\lambda(l \rightarrow \infty) \sqrt{1+\frac{\pi \xi_{0}}{2 l}} .
$$

The London penetration depth $\lambda_{L}=32 \mathrm{~nm}$ and the BCS coherence length $\xi_{0}=39 \mathrm{~nm}$ were taken once more from literature [2]. This measurement shows that the low temperature baking significantly decreases $l$, see Table II. The BCS surface resistance is only slightly altered because it has its minimum at $l=\xi_{0}[2]$.

\section{B. Field dependent surface resistance}

Figure 6 shows $R_{\mathrm{S}}$ as a function of $E_{\text {acc }}$ at $1.8 \mathrm{~K}$ for five cooldown cycles, including two after baking. At low field the surface resistance differs only by a few $\mathrm{n} \Omega$ as one can also see from Fig. 4. At larger values of $E_{\text {acc }}$ the differences in $R_{\mathrm{S}}$ become more pronounced. The lowest value is obtained for the fastest cooldown (1st cycle) before baking. Baking increases $R_{\mathrm{S}}$ significantly, especially at higher field. After baking $R_{\mathrm{S}}$ is again reduced by

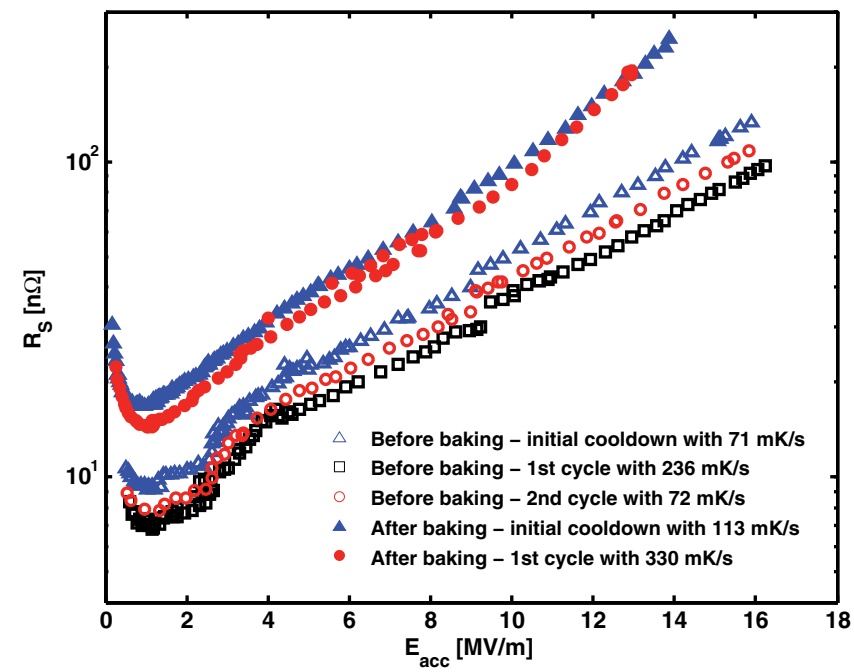

FIG. 6. Surface resistance $R_{\mathrm{S}}$ of a $1.3 \mathrm{GHz} \mathrm{Nb} / \mathrm{Cu}$ cavity measured at $1.8 \mathrm{~K}$ before (open symbols) and after baking (closed symbols) for initial cooldown and several thermal cycles above $T_{\mathrm{c}}$.

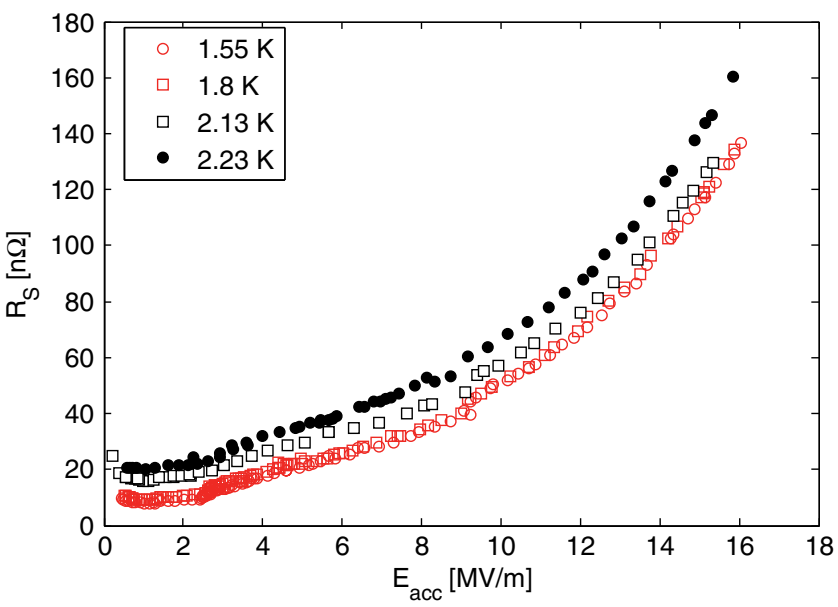

FIG. 7. Surface resistance $R_{\mathrm{S}}$ of a $1.3 \mathrm{GHz} \mathrm{Nb} / \mathrm{Cu}$ cavity measured at four different temperatures. At low field $R_{\mathrm{S}}$ is similar for the four temperatures. At higher field $R_{\mathrm{S}}$ increases more strongly if data is taken at temperatures above the lambda point, compare Table III.

fast cooldown, even though the effect is less pronounced as before.

After the initial cooldown $R_{\mathrm{S}}$ was additionally measured as a function of $E_{\text {acc }}$ for several temperatures, including one just above and one just below the lambda point of helium at $2.17 \mathrm{~K}$, see Fig. 7. One can see that at low field $R_{\mathrm{S}}$ varies only slightly for the different temperatures, which can be entirely correlated to low field BCS losses, compare with Fig. 4. For higher accelerating gradients $R_{\mathrm{S}}$ increases stronger with $E_{\text {acc }}$ for $2.23 \mathrm{~K}$ compared to the three other curves. This is the only curve measured in a normal fluid helium bath. Table III displays the increase of $R_{\mathrm{S}}$ between 1 and $15 \mathrm{MV} / \mathrm{m}$ for the four temperatures. Individual points were obtained by linear interpolation between the two data points closest to the fields of 1 and $15 \mathrm{MV} / \mathrm{m}$, respectively.

After baking and the following thermal cycle the cavity was filled with helium gas. Then $R_{\mathrm{S}}$ vs $E_{\text {acc }}$ was measured again, and also the helium pressure was recorded, see Fig. 8. In the range used the 2-digit pressure sensor had a resolution of $0.1 \times 10^{-6}$ mbar. Using the calibration obtained during the following warmup (Fig. 3) this corresponds to a minimal detectable temperature increase of $60 \mathrm{mK}$. Above $10 \mathrm{MV} / \mathrm{m}$ a pressure increase by $0.1 \times$ $10^{-6}$ mbar was detected, corresponding to a maximal temperature increase of $120 \mathrm{mK}$ within experimental error margins.

TABLE III. Surface resistance increase for four different temperatures.

\begin{tabular}{lcccc}
\hline \hline $\mathrm{T}[\mathrm{K}]$ & 1.55 & 1.8 & 2.13 & 2.23 \\
$R_{\mathrm{S}}(15 \mathrm{MV} / \mathrm{m})-R_{\mathrm{S}}(1 \mathrm{MV} / \mathrm{m})[\mathrm{n} \Omega]$ & 107.2 & 106.1 & 105.5 & 119.7 \\
\hline \hline
\end{tabular}




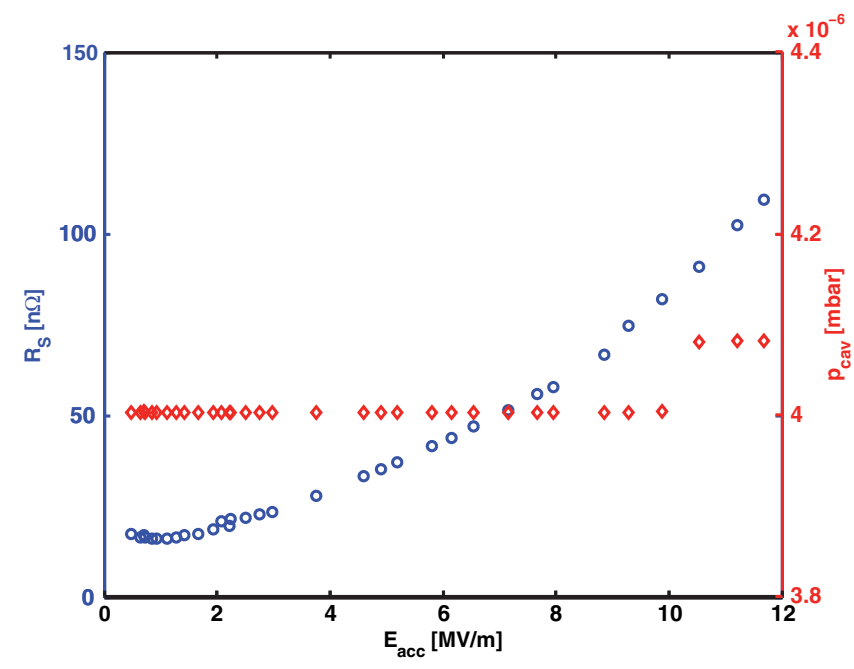

FIG. 8. Primary y-axis: Surface resistance $R_{\mathrm{S}}$ of a $1.3 \mathrm{GHz}$ $\mathrm{Nb} / \mathrm{Cu}$ cavity measured at $1.8 \mathrm{~K}$. Secondary y-axis: Helium pressure $p$ inside the cavity as a function of $E_{\text {acc }}$.

\section{DISCUSSION}

\section{A. Thermal cycling}

It was shown that fast cooling can reduce the surface resistance of elliptical $\mathrm{Nb} / \mathrm{Cu}$ cavities by about $20 \%$ at $E_{\text {acc }}=15 \mathrm{MV} / \mathrm{m}$ in a vertical test cryostat. Thermal cycling changes the low field residual resistance only slightly by fractions of a $n \Omega$. The benefit is only at higher accelerating gradients, similar to what has been observed in [9] for low temperature baked 9-cell bulk niobium cavities. However, for the $\mathrm{Nb} / \mathrm{Cu}$ cavity investigated here, this effect was reduced by baking. Furthermore, it has to be noted that the cooldown speed was at maximum $30 \mathrm{mK} / \mathrm{s}$ in [9], which is not even half as fast as the lowest cooldown speed of $70 \mathrm{mK} / \mathrm{s}$ obtained in the experiments here. In [8] it took at least one minute between the transition from the coldest to the warmest part of the cavity, here this was at maximum a few seconds. In [12] it was found by measurements using the CERN Quadrupole Resonator on a $\mathrm{Nb} / \mathrm{Cu}$ sample prepared by electron cyclotron resonance (ECR) sputtering that a fast cooldown decreases $R_{\mathrm{S}}$. The cooldown speed obtained in this experiment was comparable to what has been reported here. However it was found in contradiction to the results presented here that mainly the low field $R_{\mathrm{S}}$ is affected, while the influence on the field dependence is rather small. The results presented here suggest that the thermal gradient does not affect $R_{\mathrm{S}}$ but the cooldown speed is of importance, which is in contradiction to what has been measured on $\mathrm{Nb} / \mathrm{Cu}$ quarter wave resonators in vertical tests [13]. To conclude, it can be said that the cooldown speed and the thermal gradient play an important role for minimizing losses in superconducting cavities. Comparing results from different publications is difficult because it is still unclear whether flux trapping from ambient fields or thermal currents are more relevant. Another open question is whether cavities of different geometry and/or different materials/treatments need specific cooldown procedures. Based on the results presented here and taking into account that thermal currents might be more relevant in horizontal cryomodules than in vertical test cryostats the following procedure for horizontal SRF cryomodules equipped with $\mathrm{Nb} / \mathrm{Cu}$ cavities is advised: (i) After the initial cooldown, a warmup just above $T_{\mathrm{c}}$, (ii) Give the cavity time to thermalize to minimize thermal currents, (iii) Cooldown the cavity as fast as possible through $T_{\mathrm{c}}$. This procedure is consistent with all experimental results on $\mathrm{Nb} / \mathrm{Cu}$ cavities. In [8] it was suggested to either thermalize the cavity above $T_{\mathrm{c}}$ during the first cooldown or perform a thermal cycle. The former option is not advised for $\mathrm{Nb} / \mathrm{Cu}$ cavities. The thermalized cavity in the first initial cooldown gave an even worse result than for the slowest cycle afterwards.

\section{B. Low temperature baking}

In situ baking of the cavity yielded a higher low field residual resistance and an enhanced field dependent surface resistance, while the temperature dependent BCS surface resistance $R_{\mathrm{BCS}}$ was almost unaffected. The former two effects have also been observed for bulk niobium cavities [14]. For bulk niobium cavities $R_{\mathrm{BCS}}$ is lowered by baking, which can be correlated to a decreased mean free path $l$. An unbaked bulk niobium cavity has $l \gg \xi_{0}$. Baking pushes $l$ closer to $\xi_{0}$. For $\mathrm{Nb} / \mathrm{Cu}$ cavities baking also reduced $l$, but since these cavities already have $l \approx \xi_{0}$ before baking there is no reduction of $R_{\mathrm{BCS}}$. For bulk niobium the low temperature bake is beneficial because it shifts the exponential increase of the surface resistance referred to as $Q$-Drop to higher fields. Since the baking did not have any similar effect for the $\mathrm{Nb} / \mathrm{Cu}$ cavity it can be concluded that the origin of the field dependent surface resistance here is a different one than for the $Q$-drop of bulk niobium cavities. Currently there is no benefit from in situ baking of $\mathrm{Nb} / \mathrm{Cu}$ cavities. However, it might become useful if the medium field $Q$-slope could be understood and cured and the $Q$-drop as observed for bulk niobium cavities would become the limitation of this technology.

\section{Thermal resistance}

Measurements of $R_{\mathrm{S}}$ as a function of $E_{\text {acc }}$ just below and above $2.17 \mathrm{~K}$ the lambda point of helium have shown that the thermal resistance plays a role for the losses in $\mathrm{Nb} / \mathrm{Cu}$ cavities cooled by helium-I. At $15 \mathrm{MV} / \mathrm{m}$ the surface resistance is about $20 \mathrm{n} \Omega$ higher if the cavity is only cooled by helium-I. In helium-II however the contribution to the overall field dependent surface resistance seems to be rather low. The increase of the inner surface temperature was measured to be at maximum $120 \mathrm{mK}$ within experimental error margins. It has to be noted that for the calibration of the gas pressure with respect to the temperature the whole 
cavity, including a part of the pumping line, was warmed up and for the rf measurement only the part of the cell with high surface magnetic field. The ratio of these surface areas is roughly two. Taking this into account, the heating of the inner surface can at maximum account for an additional $R_{\mathrm{S}}$ of $6 \mathrm{n} \Omega$ assuming low field BCS losses. On the other hand, an increase of $100 \mathrm{n} \Omega$ was measured between low field and $15 \mathrm{MV} / \mathrm{m}$. It can be concluded that the thermal resistance can play an important role in minimizing the losses of superconducting cavities at high field. The thermal boundary resistance between the $\mathrm{Nb}$ film and the copper substrate is however not the main contribution for the field dependent losses of $\mathrm{Nb} / \mathrm{Cu}$ cavities. In fact, the thermal resistance should play a more important role for bulk niobium cavities. In [6] it has been reported that the overall thermal conductance of a niobium film on a copper substrate is still four times higher than of electropolished niobium. Even for bulk niobium cavities operated in heliumI it had been concluded by thermal modeling that heating of the inner surface only plays an insignificant role [25].

The method to measure the gas pressure as a function of the accelerating gradient allowed to derive the inner surface temperature and to calculate an upper estimate of how much the heating of the inner surface accounts for the field dependent surface resistance. The experiments presented here were limited by the resolution of the two digit pressure sensor and therefore only allowed to conclude that global heating is not the main contributor to the field dependent surface resistance of $\mathrm{Nb} / \mathrm{Cu}$ cavities. Further experiments, with enhanced resolution and also on bulk niobium cavities, are planned to study this effect in more detail. The method has already been tested on an LHC crab cavity and a significant pressure increase was detected. These results will be presented elsewhere. The method might also be used to obtain the quality factor $Q_{0}$ in horizontal cryomodule tests equipped with strongly coupled input antennas. In this case one could first measure $Q_{0}$ vs $E_{\text {acc }}$ and $p$ in a vertical test cryostat. After installation in the cryomodule one could insert gas in the cavity again and measure $E_{\text {acc }}$ and $p$ again and then obtain $Q_{0}$ by comparison with the results from the vertical test. This could serve as an independent method to the usual approach of obtaining $Q_{0}$ from the liquid helium consumption. It, however, has to be noted that the strong dependence of the pressure measurement on the experimental setup and the temperature distribution in the periphery need to be taken into account. Since the periphery changes entirely when moving from vertical to horizontal tests, the pressure values obtained are hard to compare. Application of this method to cryomodule testing would require a detailed study, taking into account the different volumes of the cavity with periphery in the different test setups. However, a quick uncalibrated check can always be made during helium processing, for which similar gas pressures are used.

\section{CONCLUSION}

It has been shown that a fast cooldown can significantly reduce the surface resistance of $\mathrm{Nb} / \mathrm{Cu}$ cavities. There is currently no benefit from low temperature baking for this technology. The thermal resistance plays only a minor role for the field dependent losses if the cavity is cooled by helium-II. It can be concluded that the field dependent surface resistance of $\mathrm{Nb} / \mathrm{Cu}$ is mainly limited by effects intrinsic in the film, which need to be investigated in detail if the current limitations shall be overcome.

\section{ACKNOWLEDGMENTS}

This work has been carried out in the framework of a CERN fellowship. I would like to thank Giovanni Terenziani, Serge Forel, and Damiano Sonato for preparing the cavity used in this study. Thanks for the technical support from CERN's cryolab staff for the cavity test. The fruitful discussion with my colleagues at CERN, especially Torsten Koettig, Sergio Calatroni, Giovanna Vandoni, and Sarah Aull are highly appreciated.

[1] M. Pasini, S. Calatroni, O. Capatina, A. D'Elia, M. Fraser, and M. Therasse, in HIE-ISOLDE quarter wave $\mathrm{Nb} / \mathrm{Cu}$ cavity, Proceedings of the 15th International Conference on RF Superconductivity, Chicago, IL, USA (JACoW, Geneva, 2011), p. 686.

[2] J. P. Turneaure, J. Halbritter, and H. A. Schwettman, The surface impedance of superconductors and normal conductors: The Mattis-Bardeen theory, J. Supercond. 4, 341 (1991).

[3] C. Benvenuti, S. Calatroni, I. Campisi, P. Darriulat, M. Peck, R. Russo, and A.-M. Valente, Study of the surface resistance of superconducting niobium films at $1.5 \mathrm{GHz}$, Physica (Amsterdam) 316C, 153 (1999).

[4] S. Calatroni, 20 Years of experience with the $\mathrm{Nb} / \mathrm{Cu}$ technology for superconducting cavities and perspectives for future, Physica (Amsterdam) 441C, 95 (2006).

[5] C. Benvenuti, S. Calatroni, M. Hakovirta, H. Neupert, M. Prada, and A.-M. Valente, in CERN Studies on Niobium-coated 1.5 GHz Copper Cavities, Proceedings of the 10th International Conference on RF Superconductivity, Tsukuba, Japan (JACoW, Geneva, 2001), p. 252.

[6] S. Calatroni, E. Barbero-Soto, C. Benvenuti, L. Ferreira, and $\mathrm{H}$. Neupert, in Progress of $\mathrm{Nb} / \mathrm{Cu}$ Technology with $1.5 \mathrm{GHz}$ Cavities, Proceedings of the 11th International Conference on RF Superconductivity, Luebeck/Travemnder, Germany (JACoW, Geneva, 2003), p. 544.

[7] V. Palmieri, A. A. Rossi, S. Y. Stark, and R. Vaglio, Evidence for thermal boundary resistance effects on superconducting radiofrequency cavity performances, Supercond. Sci. Technol. 27, 085004 (2014).

[8] J.-M. Vogt, O. Kugeler, and J. Knobloch, Impact of cooldown conditions at Tc on the superconducting rf cavity quality factor, Phys. Rev. ST Accel. Beams 16, 102002 (2013). 
[9] A. Romanenko, A. Grassellino, O. Melnychuk, and D. Sergatskov, Dependence of the residual surface resistance of superconducting radio frequency cavities on the cooling dynamics around Tc, J. Appl. Phys. 115, 184903 (2014).

[10] E. Mahner, S. Calatroni, E. Chiaveri, E. Haebel, and J. M. Tessier, A new instrument to measure the surface resistance of superconducting samples at $400 \mathrm{MHz}$, Rev. Sci. Instrum. 74, 3390 (2003).

[11] T. Junginger, W. Weingarten, and C. Welsch, Extension of the measurement capabilities of the quadrupole resonator, Rev. Sci. Instrum. 83, 063902 (2012).

[12] S. Aull, in Surface Resistance of a bulk-like Nb Film, The 6th International Workshop on Thin Films and New Ideas for RF Superconductivity, Legnaro, Italy (2014), http:// www.surfacetreatments.it/thinfilms-2014/.

[13] P. Zhang, in The influence of cooldown conditions at transition temperature on the quality factor of niobium sputtered quarter-wave resonators, The 6th International Workshop on Thin Films and New Ideas for RF Superconductivity, Legnaro, Italy (2014), http://www .surfacetreatments.it/thinfilms-2014/.

[14] G. Ciovati, Effect of low-temperature baking on the radiofrequency properties of niobium superconducting cavities for particle accelerators, J. Appl. Phys. 96, 1591 (2004).

[15] B. Aune et al., Superconducting TESLA cavities, Phys. Rev. ST Accel. Beams 3, 092001 (2000).

[16] G. Terenziani, S. Calatroni, T. Junginger, I. Santillana, and A. Ehiasarian, in $\mathrm{Nb}$ coating developments with HIPIMS for SRF applications, Proceedings of the 16th International Conference on RF Superconductivity, Paris, France (JACoW, Geneva, 2013).
[17] F. Magnus, A. S. Ingason, O. B. Sveinsson, S. Olafsson, and J. T. Gudmundsson, Morphology of TiN thin films grown on $\mathrm{SiO} 2$ by reactive high power impulse magnetron sputtering, Thin Solid Films 520, 1621 (2011).

[18] G. Terenziani, private communication, Ph.D. thesis, University of Sheffield (in preparation).

[19] H. Padamsee, T. Hays, and J. Knobloch, RF superconductivity for Accelerators (Wiley, New York, 2008), 2nd ed., Vol. 577.

[20] R. H. Carr, R. D. McCammon, and G. K. White, The Thermal Expansion of Copper at Low Temperatures, Proc. R. Soc. A 280, 72 (1964).

[21] C. Benvenuti, in Recent Advances in Vacuum Techniques for Accelerators, Proceedings of the 10th International Conference on Cyclotrons and their Applications, East Lansing, Michigan, USA (JACoW, Geneva,1984), p. 540.

[22] G. Ciovati (private communication); J. Halbritter, Kernforschungszentrum, Karlsruhe, Externer Bericht Report No. 3/69-2, 1969; Kernforschungszentrum, Karlsruhe, Externer Bericht Report No. 3/70-6, 1970; J. Halbritter, Z. Phys. 266, 209 (1974).

[23] C. J. Gorter and H. Casimir, On supraconductivity I, Physica (Amsterdam) 1, 306 (1934).

[24] A. B. Pippard, An Experimental and Theoretical Study of the Relation between Magnetic Field and Current in a Superconductor, Proc. R. Soc. A 216, 547 (1953).

[25] R. L. Geng and H. Padamsee, On the low-field Q-slope of RF superconducting niobium cavities cooled by helium-I, Cornell University LNS report, Report No. SRF01121210, 2001. 